\title{
MEASURING PAIN BEHAVIOUR: SCALE OF PAIN EXPRESSION BEHAVIOURS (SOPEB)
}

\author{
Simmy M. Varkey ${ }^{1 *}$, PK Babu ${ }^{2}$, Varghese P. Punnoose ${ }^{3}$ \\ ${ }^{1}$ Ph.D. Scholar, Dept. of Behavioural Sciences, MG University, Kottayam. \\ ${ }^{2}$ Associate Professor of Statistics, Dept. of Community Medicine, Government Medical College, Manjery. \\ ${ }^{3}$ Professorand Head, Dept. of Psychiatry, Government Medical College, Alappuzha. \\ *Correspondence: Dept. of Behavioural Sciences, M.G University, Kottayam. E-mail: simmyscariah@gmail.com
}

\begin{abstract}
Nonverbal communication refers to how people communicate, intentionally or unintentionally, without words. A study was conducted in general and taluk hospitals of Kottayam to develop a scale to measure pain behaviour: Scale of Pain Expression Behaviours (SOPEB). Consecutive patients brought to the hospitals with diabetic foot ulcer $(n=350)$ were given SOPEB. They were also given Adult Nonverbal Pain Scale. We determined content validity index. Construct validity for SOPEB was computed through exploratory factor analysis. These factors concentrate and index the dispersed information in the original data and replace 105 characteristics to 25 characteristics in each domain. Kaiser-Meyer-Olkin Test values was 0.602 , and it determines suitability of data for factor analysis. After rotation, it identified five linear components which gave eigenvalue of more than 1. A Scree Plot was used to visually assess which components or factors explain most of the variability in the data. Concurrent validity is used to correlate SOPEB scores with adult Nonverbal Pain Scale. The reliability of each item was calculated using percent agreement and the Cohen's Kappa coefficient. Nonverbal communication has a key role in patient care. Effective communication takes into account nonverbal communication also. Usage of tools such as SOPEB helps to assess the proper quantification of pain behaviour.
\end{abstract}

Keywords: pain, nonverbal communication, diabetic foot

Nonverbal behaviour is a broad term that refers to all the communicative actions and behaviours that we can engage which do not involve words. Nonverbal behaviour generally communicates through three "channels": facial expressions, vocalisations, and body postures. ${ }^{1}$ In a communication, $7 \%$ of the message comes from words and the other $93 \%$ comes from nonverbal signals, according to Albert Mehrabian's communications model. ${ }^{2}$ CarisVerhallen et al. (1999) had experimented and analysed the data in hierarchical linear models to understand the relationship between nonverbal and verbal communication. ${ }^{3}$

\section{CLINICAL UTILITY AND POTENTIAL USES OF THE SCALE}

Pain is reliably expressed in the face, although culture and context modify expressiveness. However, facial expression requires more detailed assessment than which is usually practiced, because it may be the only unique indicator of pain in people with compromised or underdeveloped communication capacities or whose personality may not vocalise their pain. Other 
motor behaviours, particularly guarding, are of interest and can be assessed by observation, preferable to self-reporting of that behaviour. ${ }^{4}$

It is not necessary to have a verbal acknowledgement of pain to recognise it; instead, health personnel can use the behaviour cues to identify whether a patient is in the midst of a painful experience. ${ }^{5}$ Facial expressions of pain are well documented and generally take the form of a grimace, with teeth bared and eyes tight. Vocalisations, such as crying out, have also been found to communicate pain to listeners. There is also a lot of work which has looked into how pain influences our body posture, how we stand and move when in pain. There are pain behaviours, such as protectively holding injury sites and moving more cautiously, and these are well documented, but up until now, very little research has considered whether these behaviours communicate information to observers. $^{6}$

Block et al. (1980) studied 20 married chronic pain patients and found that the responses of their spouses to pain behaviour was less. This may be a reason for them reporting marginally higher levels of pain in the spouse-observing condition than in the neutralobserver condition. ${ }^{7}$

Darwin's research on facial expressions has had a major impact on the field in many areas; all human beings everywhere encode or express these emotions in the same way, and all human beings can decode or interpret them with equal accuracy. ${ }^{8}$ Haidt $\&$ Keltner (1999) concluded that a distinctive expression of embarrassment existed, composed of turning the head away, looking down, shifting the gaze to the side, evincing a controlled smile (e.g., with the lips pressed), and sometime, touching the face with one's hand — all lasting up to five seconds in a typical instance. ${ }^{9}$

Without a doubt, the crown jewel of nonverbal communication is the facial expressions channel. This aspect of communication has the longest history of research, beginning with Charles Darwin's (1872) book "The Expression of the Emotion in Man and Animals". ${ }^{10}$ Lundberg et al. note that display rules are particular to each culture and dictate what kind of emotional expression people are supposed to show. ${ }^{11}$
For example, American cultural norms discourage emotional displays in men, such as grief or crying, but allow the facial display of such emotions in women. In Japan, conventional cultural rules dictate that women should not exhibit a wide, uninhibited smile; Japanese women will often hide their wide smiles behind their hands while Western women are allowed — indeed encouraged — to smile broadly and often. In fact, the cultural display rules that govern Japanese nonverbal expression are surprisingly different from Western ones, Japanese norms lead people to cover up negative facial expressions with smiles and laughter and to display fewer facial expressions in general than is true in the West. This is undoubtedly what lies behind the Western stereotype that Asians are "inscrutable" and "hard to read". ${ }^{12}$ Many forms of nonverbal behaviour are specific to a given culture. Not only do some of the nonverbal behaviours of one culture mean nothing in another, the same nonverbal behaviours can exist in two cultures but have very different meaning in each. ${ }^{13}$ The ability to recognise the pain of others with a high degree of accuracy underpins our ability to get others to help us; the point of any pain communication is, at least in part, to encourage others to help us, and it seems that body postures and pain behaviours are an effective way to get this message across. ${ }^{14}$

Pain can be communicated nonverbally through facial expressions, vocalisations, and bodily movements. Most studies have focussed on the facial display of pain, whereas there is little research on postural display. Stimulus sets for facial and vocal expressions of pain have been developed, but there was no equivalent for body-based expressions. ${ }^{15}$ Pain and disgust are facial expressions which share many similarities. Both are negative expressions, and they both share similar origins, and because of this, share some similarities in their nonverbal expression. ${ }^{16}$

In many clinical and experimental settings, the facial expression of pain is incorporated with verbal and nonverbal vocal activity, posture, and movement in an overall category of pain behaviour. This is assumed by clinicians to be under operant control of social contingencies such as sympathy, caregiving, and practical help. Thus, a strong facial expression is 
presumed to constitute an attempt to manipulate these contingencies by amplification of the normal expression. ${ }^{17}$

Scale of Pain Expression Behaviours (SOPEB) is a tool developed and standardized by us for measuring pain behaviour during debridement and dressing of chronic wounds. It requires recording of each pain behaviour as it occurs into smaller units of observation using a molecular approach. Most often, patients exhibit severe pain during procedures such as wound debridement and dressing changes, and many have anticipatory fear to undergo them. This tool tends to be useful clinically as well as for research purposes because of its ability to ascertain grade of pain during painful procedures such as debridement and to discriminate among pain events when the health personnel is unable to perform a verbal interview.

\section{MATERIALS AND METHODS}

The study was conducted in general and taluk hospitals of Kottayam district which is under the jurisdiction of Directorate of Health Services (DHS). Official permission was obtained from DHS. Consecutive patients who were brought to the hospitals with diabetic foot ulcer within a period of one year were taken up for the study. Informed consent from the patients was taken. SOPEB was used on 350 consecutive diabetic foot ulcer patients admitted in six different taluk and general hospitals of Kottayam district on three consecutive debridement days. They were also assessed with adult Nonverbal Pain Scale (NVPS) by University of Rochester Medical Centre. The NVPS is a fiveitem pain scale measuring the face, activity/movement, guarding, vital signs, and respirations rated on a Likert scale. Scores can range from 0 to 10, with lower scores indicating less pain. Scores of 0-2 indicate no pain, 3-6 moderate pain, and 7-10 severe pain. Presence of behaviours during the procedure was observed by the researcher using SOPEB on three days of debridement and was coded using scores assigned to the presence of each item as always-5, most of time-4, never-3, sometimes-2, rarely-1.

\section{Psychometric Properties}

\section{Assessment of content validity}

Ten clinical nurses and 10 surgeons were interviewed in order to generate a list of pain-related behaviours that patients usually exhibited during debridement of diabetic foot wounds or burns dressing. Based on their reports and clinical experience of the researchers, items of pain expression were developed. As a first step in evaluating validity, we chose valid items from the constructed ones. From the various items initially considered for the scale, the final version was agreed through consensus method following discussion among faculties. A multidisciplinary panel of nine judges was given the tool to determine the clarity, relevance, simplicity, and appearance of each question. We determined the agreement between the experts using content validity index. Content validity ratio was also computed using Lawshe's formula. Those items which gained CVI $>.80$ were chosen for the final tool.

\section{Assessment of construct validity}

Construct validity is the degree to which the tool conforms to the predicted correlations with other theoretical propositions. Construct validity for SOPEB was computed through exploratory factor analysis (EFA) using SPSS 20. Factor analysis was employed to discover the basic structure of domains of pain expression behaviour by tracing hidden patterns and their overlap. It is used to create a set of variables for similar items in the set called dimensions. Data reductions of 105 characteristics for 350 patients would be unwieldy to handle descriptively or analytically. The management, analysis, and understanding of such data are facilitated by reducing them to their common factor patterns. These factors concentrate and index the dispersed information in the original data and can, therefore, replace 105 characteristics to five characteristics in each domain without much loss of information. We viewed the pattern of variation among pain behaviours across their characteristics and then grouped the pain behaviours by their profile similarity. In some domains, more than five variables were present. After considering the clinical 
perspective, the number was brought down to five per domain.

Pain behaviours high on facial expression domain also appear low on treatment refusal domain. There is a regularity of patterns, therefore, in the pain behaviours on these five domains, and this regularity is described as a pattern of variation. The factors that explain the least amount of variance are generally discarded.

\section{RESULTS}

Kaiser-Meyer-Olkin (KMO) Test values were 0.602 (Table 1). This test determines the suitability of data for factor analysis. Initial eigenvalues were computed. Factor extraction sums of Squared Loadings and rotation sums of Squared Loadings were done. Eigenvalues associated with each factor before extraction, after extraction, and after rotation. After rotation, it identified five linear components which gave eigenvalue of more than 1 . The first five components had an eigenvalue that exceeds the criterion value of 1 . Then, $80 \%$ rule was applied which helps to retain those components which could explain at least $80 \%$ of the total variance. Here also, the above five components together accounted for $80 \%$ of the total variability.

A Scree Plot was used to visually assess which components or factors explain most of the variability in the data and to depict the descending variances that account for the factors extracted in graph form (Figure 1). The factors that lie before the point at which eigenvalues begin to drop can be retained. The recommendation is to retain all components with eigenvalues in the sharp descent of the line before the first one where the levelling effect occurs. After correlating with criteria 1 and 2, it was decided to include only five, as from point six onwards the sharpness started to disappear.

This scree plot also shows that five of those factors already identified explain most of the variability because the line starts to straighten after factor 5 .
Table 1: KMO and Bartlett's test

\begin{tabular}{|c|c|c|}
\hline \multicolumn{3}{|c|}{ KMO and Bartlett's test } \\
\hline \multicolumn{2}{|c|}{$\begin{array}{l}\text { Kaiser-Meyer Olkin Measure of } \\
\text { sampling adequacy }\end{array}$} & .602 \\
\hline \multirow{3}{*}{$\begin{array}{l}\text { Bartlett's test } \\
\text { of sphericity }\end{array}$} & Chi-Square & 2164.359 \\
\hline & df & 66 \\
\hline & Significance & $<0.0001$ \\
\hline
\end{tabular}

Figure 1: Scree Plot

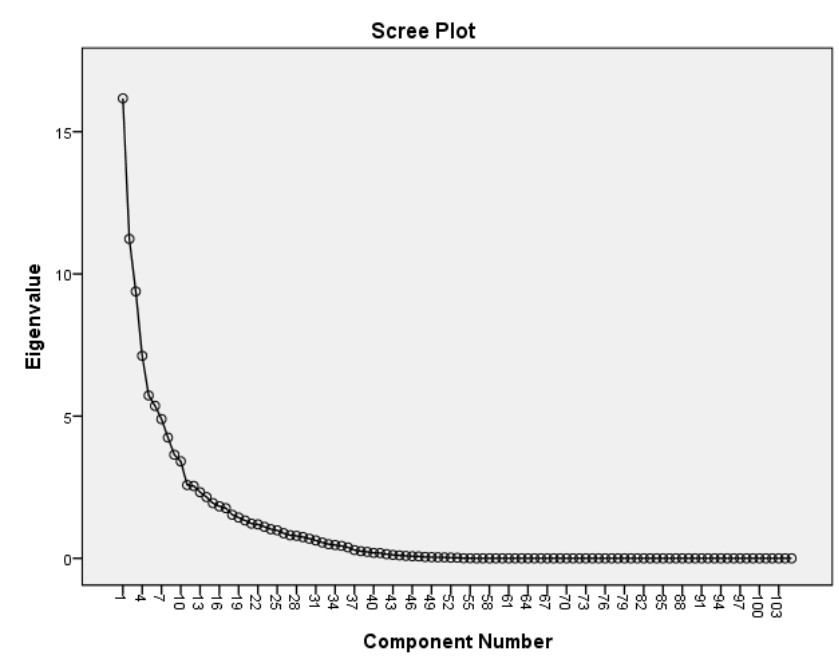

Domains were identified as follows:

Facial expression domain $\quad-25$ items

Physical symptom domain $\quad-25$ items

Treatment refusal domain $\quad-25$ items

Vocalisation domain $\quad-25$ items

Tense body language domain -25 items

The remaining factors explain a very small proportion of the variability and are likely to be unimportant.

\section{Assessment of criterion-related validity}

Criterion-related validity compares constructed test to another test measuring close to evaluated measure which had accepted psychometric 
properties. The correlation between the scores of the two tests serves as the validity coefficient. Both tests are administered simultaneously within the same general time frame. Concurrent validity is used to correlate SOPEB scores with Adult nonverbal pain scale University of Rochester Medical Center. The scores of SOPEB showed a high correlation with NVPS (0.925; $\mathrm{P}<0.01)$.

\section{Assessment of inter-rater reliability}

Since the raters will agree about $50 \%$ of the times, we used a sample size of 100 . Two independent nurse observers assessed 100 diabetic foot patients undergoing debridement using the SOPEB Checklist. The reliability of each item was calculated using percent agreement and the Cohen's Kappa coefficient. Total percent agreement ranged from $90 \%$ to $100 \%$, and Kappa statistics ranged from 0.80 to 1.00 . Study findings confirmed that the present study is using a reliable tool for identifying the pain expression behaviours.

\section{SCORING OF SOPEB}

A tick mark indicating the presence of pain expression behaviour is given the score of 1 , and a cross mark for its absence is given the score of 0 in each column containing statements of behaviour. Add up the scores in each row to find out item score in the scoring column on right side of statements. Add up the cumulative scores on each row to find out the total score. Each domain is scored out of 25. Individual domains are measured according to the quartile range as 0 ("no pain") mild (1-8), moderate (9-15) and severe scores (16-25). Total scores according to the quartile range are classified into no pain (0), mild (1-42), moderate (43-82) and severe (83-125).

Nonverbal communication has a key role in patient care. Effective communication takes into account nonverbal communication also. Usage of tools such as SOPEB helps to assess the proper quantification of pain behavior.

\section{DISCUSSION}

One important way for communication of pain is non-verbal behaviour. Previous non-verbal scales for measuring pain was not including all the possible domains. Nonverbal behaviours observed in those previous scales were patient-directed eye gaze, affirmative head nodding, smiling, forward-leaning, affective touch, instrumental touch and autonomic behaviour changes. The results demonstrated that health care providers use mainly eye gaze, head nodding and smiling to establish a good relationship with their patients. The use of affective touch is mainly attributable to one's personal style. Nonverbal forms of communication have typically been studied individually, in their separate "channels" (e.g., eye gaze or gestures), even though, in everyday life, nonverbal cues of many kinds occur, all at the same time in a quite dazzling orchestration of information. Nonverbal cues serve many functions in communication. SOPEB would be useful for measuring pain behaviour during debridement and dressing of chronic wounds. It facilitates smaller units of observation, conveying pain behaviours and facilitating verbal communication.

\section{REFERENCES}

1. Knapp ML, Daly JA, editors. Handbook of interpersonal communication. California: SAGE Publications; 2002.

2. Albert M. Silent Messages. 1st ed. Belmont(CA): Wadsworth; 1971.

3. Caris-Verhallen WM, Kerkstra A, Bensing JM. Nonverbal behaviour in nurse-elderly patient communication. J AdvNurs 1999; 29:808-18.

4. Ashraf AB, Lucey S, Chen T, Cohn JF, Ambadar Z, Prkachin KM, et al. The painful face - pain expression recognition using active appearance models. Image Vis Comput 2009; 27:1788-96.

5. Turk DC, Meichenbaum D, Genest M. Pain and behavioral medicine: a cognitive-behavioral perspective. New York: Guilford Press; 1983.

6. Sinha N. Self-other rating discrepancy and personality antecedents [monograph on the internet]. Ann Arbor: ProQuest ETD Collection for FIU; 2003 [cited 2018 Feb 27]. Available from: http://digitalcommons.fiu.edu/ dissertations/AAI3125051

7. Block AR, Kremer EF, Gaylor M. Behavioral treatment of chronic pain: The spouse as a discriminative cue for pain behavior. Pain 1980; 9:243-52. 
8. Harrigan JA, O'Connell DM. How do you look when feeling anxious? Facial displays of anxiety. Pers Individ Dif 1996; 21:205-12.

9. Haidt J, Keltner D. Culture and facial expression: openended methods find more expressions and a gradient of recognition. Cogn Emot 1999; 13:225-66.

10. Darwin C, Ekman P. The expression of the emotions in man and animals. 4th ed. Oxford University Press.

11. Lundberg M, Grimby-Ekman A, Verbunt J, Simmonds MJ. Pain-related fear: a critical review of the related measures. Pain Research and Treatment [serial on the jul 29]; 2011(494196): [about 26 p.]. Available from https://www.hindawi.com/journals/prt/2011/494196 /cta/

12. Kappas A. The fascination with faces: are they windows to our soul? J Nonverbal Behav 1997; 21:157-61.

13. Johnson-Laird PN, Oatley K. Basic emotions, rationality, and folk theory. Cogn \& Emot 1992; 6:20123.

14. Simon D, Craig KD, Gosselin F, Belin P, Rainville P. Recognition and discrimination of prototypical dynamic expressions of pain and emotions. Pain 2008; 135:55-64.

15. Walsh J, Eccleston C, Keogh E. Pain communication through body posture: the development and validation of a stimulus set. Pain 2014; 155:2282-90.

16. Cameron AD. (2011). Personality's interaction with the pain experience. Available from http://etheses.bham.ac.uk/1557/.

17. Prkachin, KM. Assessing pain by facial expression: facial expression as nexus. Pain Res Manag 2009; 14:53-8.

Source of support: None

Conflict of interest: None declared

First submitted: 23 ${ }^{\text {rd }}$ December 2017

Published online: $31^{\text {st }}$ March 2018 